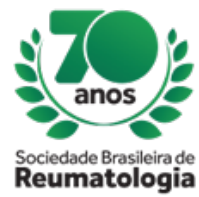

\title{
ANALYSIS OF RETENTION RATE, ADVERSE EFFECTS AND PREDICTORS OF IMUNOBIOLOGICAL THERAPY ON 15 YEARS
}

Claudia Camargo (HUCFF, rio de janeiro, RJ, Brasil), Marcia Ramos-e-Silva (HUCFF, rio de janeiro, RJ, Brasil), Sueli Carneiro (HUCFF, rio de janeiro, RJ, Brasil)

\section{BACKGROUND}

Immunobiologicals began to be used a little over a decade ago for psoriasis in moderate to severe forms. The persistence of immunobiologicals in the treatment of chronic dermatological diseases has been studied in recent years. All of the predictive factors that led to discontinuation were not identified. This study examined the persistence rate of Etanercept, Adalimumab, Ustequinumab, and Infliximab and discussed the adverse events reported by patients with psoriatic disease treated at the dermatology outpatient clinic and verified its clinical implications in this population study in the 15 -year period

\section{MATERIALS AND METHODS}

retrospective, longitudinal study conducted at the Dermatology outpatient clinic including patients who used immunobiologicals: from 2003 to 2017 for moderate to severe psoriatic disease

\section{RESULTS}

78 patients were evaluated. The prevalence of medication use was $49.5 \%$ for Etanercept, $31.2 \%$ for Adalimumab, $14 \%$ for Infliximab and $5.4 \%$ for Ustequinumabe ( Figure 1)The adverse events observed were all reported in the literature and described in the literature, with the prevalence of local injection reaction (27\%), labyrinth disease (16\%), and infection and infestation (14\%). There were 2 pregnancies in use of therapy and 1 death. Patients who used the drug Infliximab have 3.75 times the risk of discontinuing treatment when compared to patients who took the drug Etanercept. No more significant results were found in the comparison between the drugs.

\section{CONCLUSION}

Immunobiological therapy has revolutionized the treatment of moderate to severe psoriasis disease, but although widely used, there is no protocol of choice for the medication, nor sedimented knowledge of its positive predictors or discontinuation.

It was possible to demonstrate a 3.75 -fold higher risk of discontinuation of Infliximab compared to Etanercept,(Figure 2) which had the highest persistence rate at 14 years, of $57 \%$. Adverse events presented by patients during therapy, comorbidities and other possible predictors of therapy such as sex, age, age of onset of illness, associated arthritis, first immunobiological therapy, and no statistically significant data were found, but are consistent with many data of the last studies, being very useful to guide new studies on these drugs in daily clinical practice. 